\title{
Impact of dance therapy on quality of life among patients with mental illness
}

\author{
Sampoornam. $\mathbf{W}^{* 1}$, Devanbu. $\mathbf{N}^{2}$ \\ 1 Associate Professor, Mental health nursing department, The Tamilnadu Dr.M.G.R. Medical University, Chennai, Dhanvantri College of \\ Nursing Pallakkapalayam, Namakkal. Tamilnadu, India \\ 2 Dhanvantri College of Nursing Pallakkapalayam, Namakkal, Tamilnadu, India \\ *Corresponding Author: Dr. Sampoornam. W, Associate Professor, Mental health nursing department, The Tamilnadu Dr.M.G.R. \\ Medical University, Chennai, Dhanvantri College of Nursing Pallakkapalayam, Namakkal. Tamilnadu, India, Email: \\ sampoornamwebster@yahoo.in
}

Received: January 09, 2019; Published: March 28, 2019

\section{Abstract}

Background: Literally mentally ill patients are impotent and not precise in expressing their quality of life. In order to rectify the practical complexity of assessing the quality of life, modified brief psychiatric rating scale was adopted in this study to select mentally ill patients with mild level of psychiatric symptoms. Aim: This article focus on the impact of dance therapy on quality of life among patients with mental illness. Methods: Quasi experimental research design was selected for the present study. Purposive sampling technique was used to recruit 30 subjects, out of which 20 subjects in experimental group and 10 in control group. Pre test was conducted by using modified quality of life assessment scale in experimental and control group. Dance therapy was given by adopting alternative steps which involves both extremities. Each session was carried out for 20 minutes per day for 15 days. Control group received standard methods of care. Post test was conducted by using the same assessment tools. Results: The estimated paired ' $\mathrm{t}$ ' test value showed statistical significance $t=8.73$ in experimental group. Un paired ' $\mathrm{t}$ ' test value depicted marginal statistical significance $\mathrm{t}=2.78$.

Keywords: Dance therapy, Quality of life, Mental illness, Schizophrenia, Depression and Alcohol Dependence.

\section{INTRODUCTION}

Quality of life (QOL) is the general well-being of individuals and societies, outlining negative and positive features of life [1]. According to the World Health Organization (WHO), quality of life is defined as "the individual's perception of their position in life in the context of the culture and value systems in which they live and in relation to their goals [2].

Dance therapy or dance movement therapy (DMT) is defined as the psychotherapeutic use of movement as a process which furthers the emotional, social, cognitive, and physical integration of the individual. It may be of value for people with developmental, medical, social, physical or psychological impairments. Dance therapy can be practiced in mental health rehabilitation units, nursing homes, day care centres and incorporated into disease prevention and health promotion programmes [3].

The evidence shows that dance movement therapy may have an effect on reducing psychiatric symptoms and improve well- being. Overall, dance movement therapy was an effective treatment technique for mental illnesses [4].

\section{MATERIALS AND METHODS}

After obtaining informed assent, mentally ill patients who were clinically diagnosed with schizophrenia, depression and alcohol dependence \& fulfilled the inclusion criteria were enrolled in the study at Government Head Quarters Hospital, Erode. Subjects who suffered from mania, epilepsy and mental retardation were excluded from the research work.

Quasi experimental research design was selected for the present study. Purposive sampling technique was used to recruit 30 subjects, out of which 20 subjects in experimental group and 10 in control group. In order to rectify the practical complexity of assessing the quality of life, modified brief psychiatric rating scale was adopted in this study to select mentally ill patients with mild level of psychiatric symptoms.

Demographic data like age, gender, past history of hospitalization, family history of mental illness, taking medication 
and duration of illness were collected from the subjects and condensed. Pre test was conducted by using modified quality of life assessment scale in experimental and control group. Dance therapy was given by adopting alternative steps which involves both extremities. Each session was carried out for 20 minutes per day for 15 days. Control group received standard methods of care. Post test was conducted by using the same assessment tools. Statistical methods adopted were mean, standard deviation, paired ' $t$ ' test, Un paired 't' test and chi square test with Yates correction.

\section{RESULTS}

In post test $85 \%$ of the subjects had pretty good level of quality of life in experimental group, whereas control group showed $60 \%$ of the subjects had pretty bad level of quality of life (table 1 ). experimental group showed 12 and posttest showed 22 . Likewise, comparison of mean of pretest quality of life scores in control group showed 20.5 and the estimated posttest also portrayed the same value. The estimated paired ' $t$ ' test value showed statistical significance $t=8.73$ in experimental group, whereas control group delineated statistical non significance $t=$ 1.40. Estimated un paired ' $t$ ' test value depicted marginal statistical significance $t=2.78$, when compared with the mean of control group (Mean=20.5) experimental group mean score was high (Mean $=22$ ) which indicates that quality of life has been enhanced in the experimental group (Table-2).

No significant association was observed between posttest quality of life scores for age, gender, past history of hospitalization, family history of mental illness, taking medication and duration of illness in both experimental \& control group.

Comparison of mean of pretest quality of life scores in

Table 1: Frequency and percentage distribution of level of quality of life among patients with mental illness

\begin{tabular}{|c|c|c|c|c|c|c|c|c|}
\hline \multirow[t]{3}{*}{ Level of quality of life } & \multicolumn{4}{|c|}{ Experimental group } & \multicolumn{4}{|c|}{ Control group } \\
\hline & \multicolumn{2}{|c|}{ Pre test } & \multicolumn{2}{|c|}{ Post test } & \multicolumn{2}{|c|}{ Pre test } & \multicolumn{2}{|c|}{ Post test } \\
\hline & $\mathrm{F}$ & $\%$ & $\mathrm{~F}$ & $\%$ & $\mathrm{~F}$ & $\%$ & $\mathrm{~F}$ & $\%$ \\
\hline Very well & - & - & 3 & $15 \%$ & - & - & - & - \\
\hline Pretty good & - & - & 17 & $85 \%$ & - & - & - & - \\
\hline Good \& bad & 7 & $35 \%$ & - & - & 4 & $40 \%$ & 4 & $40 \%$ \\
\hline Pretty bad & 13 & $65 \%$ & - & - & 6 & $60 \%$ & 6 & $60 \%$ \\
\hline
\end{tabular}

Table 2: Mean, standard deviation, paired 't' test \& un paired ' $\mathrm{t}$ ' test value on quality of life in course of pre and post test scores in experimental and control group

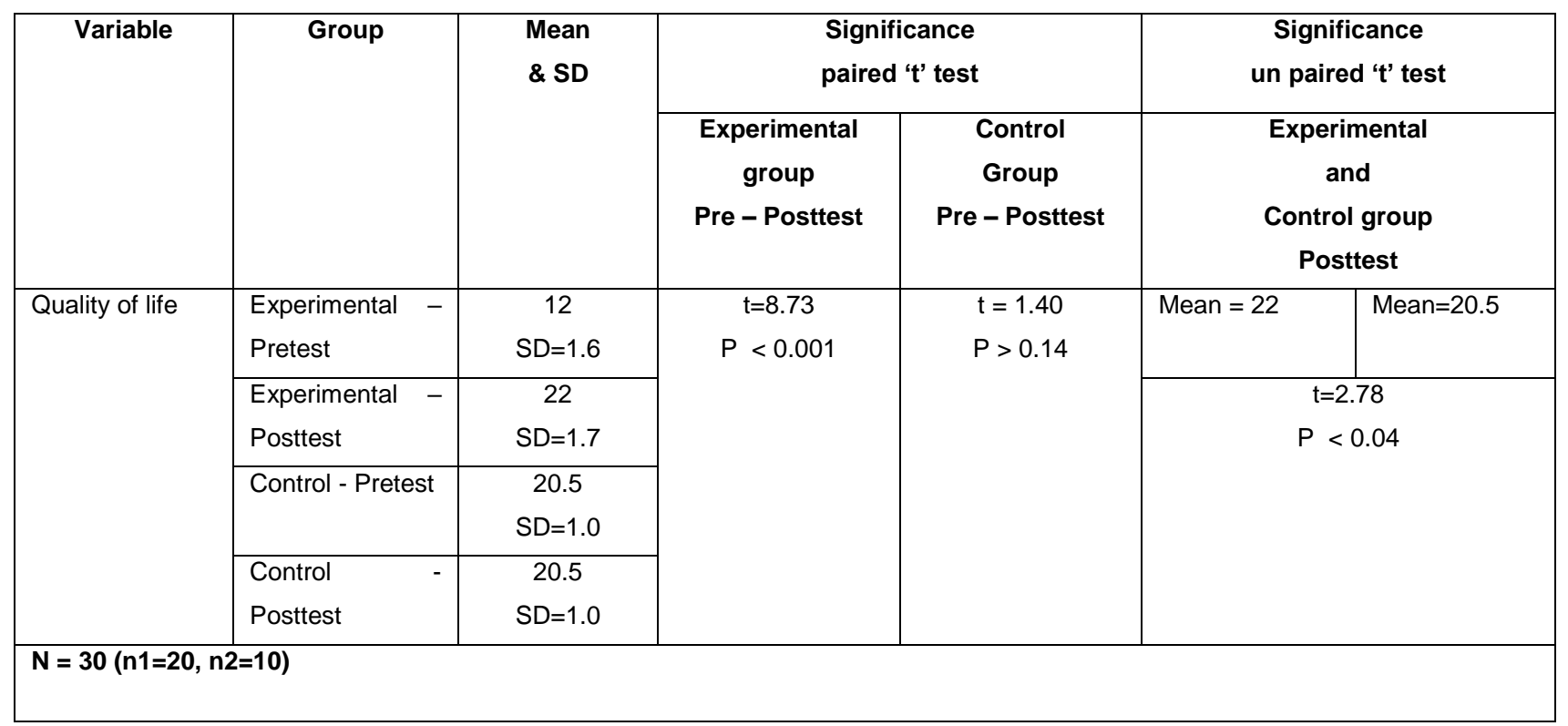

\section{DISCUSSION}

This study signifies that dance therapy is effective in improving the quality of life among patients with mental illness. Conferring to another study, dance movement therapy improved wellbeing, mood and affect, quality of life, body image and interpersonal competence and reduced the symptoms of anxiety and depression [5].
Another study investigated the effect of adding dance/movement group therapy (DMT) to the treatment of psychiatric outpatients with a diagnosis of depression. Compared to the treatment as usual (TAU), adding DMT seemed to improve the effect of the treatment. The results indicated that DMT is beneficial in the treatment of depressed patients [6]. Dance has one impact on health that explains its' benefits for mental health. Dance increases the endorphin levels, which provides elevated mood [7]. 


\section{Conflict of Interest}

None declared

\section{Financial Support}

None declared

\section{REFERENCES}

1. Barcaccia, Barbara. "Quality Of Life: Everyone Wants It, But What Is It?". Forbes/ Education. 2013.

2. McNally, James W. Encyclopedia of the Life Course and Human Development. 2009. (vol.3 ed.). USA: Macmillan Reference. p. 317.

3. Ren J, Xia J. Dance therapy for schizophrenia. Cochrane Database Syst Rev. 2013 Oct 4;(10).

4. Buse, Zeynep, Sarikaya, Zeynep, Colucci, Erminia. The Effectiveness of Dance Movement Therapy (DMT) on Reducing Symptoms of Mental Illness: A Systematic Review. Thesis. 2017.

5. Koch, S., Kunz, T., Lykou, S., \& Cruz, R. Effects of dance movement therapy and dance on health-related psychological outcomes: A meta-analysis. The Arts in Psychotherapy, 2014: 41, 46-64.

6. Paivi M. Pylvanainen, A dance movement therapy group for depressed adult patients in a psychiatric outpatient clinic: effects of the treatment. Front Psychol. 2015; 6: 980.

7. Alpert, P. T. The health benefits of dance. Home Health Care Management \& Practice, 2011: 23(2), 155-157. 